Setting Lower Limits High: The Role of Boundary Goals

in Achievement Motivation

\author{
Katherine S. Corker \\ M. Brent Donnellan \\ Michigan State University
}

Accepted for publication in Journal of Educational Psychology on October 7, 2011.

Acknowledgements: The authors thank Joseph Cesario and Kevin Ford for their helpful comments and Sara Jelinek, Kimberly Pingatore, and Jenna Kuhn for their assistance with data collection. Correspondence concerning this article should be addressed to Katherine S. Corker, Psychology Building, Michigan State University, East Lansing, MI 48824. E-mail:

corkerka@msu.edu

Abstract: Boundary goals specify the minimum performance level that an individual must attain to subjectively experience success. The current research integrates boundary goals into the hierarchical model of achievement motivation (A. Elliot, 2006) by positing that boundary goals are a sub-goal in the goal hierarchy. We predicted that performance approach goals would be associated with higher boundary goals, whereas performance avoidance goals would be associated with lower boundary goals. We further predicted that boundary goals would mediate the association between achievement goals and performance, independent of other target goals (i.e., levels of aspiration). We also evaluated whether boundary goals served a similar role in explaining associations between mastery goals and performance. We tested these predictions by tracking the performance of 347 college students across the semester. As predicted, performance approach goals were positively associated with boundary goals $(\beta=.32)$ whereas performance avoidance goals were negatively associated with boundary goals $(\beta=-.11)$. Further, we found that mastery approach goals had positive associations with boundary goals $(\beta=.29)$ whereas the opposite pattern occurred for mastery avoidance goals $(\beta=-.25)$. Boundary goals were positively linked to exam scores $(\beta=.32)$ and mediated the associations between performance approach, mastery approach, and mastery avoidance goals and grades. These statistical effects were independent of the effects of level of aspiration. In short, boundary goals seem to play an important role in the achievement motivation process and may therefore serve as a potentially useful focus for interventions.

Keywords: Achievement goals, goal orientation, academic achievement, motivation 


\section{Setting Lower Limits High: The Role of Boundary Goals in Achievement Motivation}

"The greatest danger for most of us lies not in setting our aim too high and falling short but in setting our aim too low and achieving our mark." - Michelangelo

Target goals - an individual's specific standards for performance (Harackiewicz \& Sansone, 1991) - play an important role in self-regulation and achievement. Whereas classic goal researchers have long been interested in the role of target goals in goal pursuit (e.g., Lewin's concept of level of aspiration), contemporary achievement goal researchers have focused more on underlying motivational concerns than target goals per se. The current research is an attempt to fill this gap by integrating relatively broad achievement goal orientations with target goals. We also investigate the association of a specific type of target goal, boundary goals, with academic performance. Boundary goals are the minimum performance level that one must attain to subjectively experience success. We believe that boundary goals are an important kind of target goal that are particularly relevant for performance in educational settings such as college courses. Indeed, many students may have general aspirations of obtaining high scores yet differ in terms of their minimum standards for acceptable performance. Thus, boundary goals may have something unique to contribute to understanding performance beyond high aspirations. Accordingly in the present research, we evaluate whether boundary goals and level of aspriation are predictive of achievement outcomes in a college classroom.

We conceptualize boundary goals as an important "bottom line" standard for judging levels of performance acceptable to an individual. For some students, only the highest possible level of achievement is indicative of success (i.e., boundary goal = a grade point average of 4.0), but for other students, lower marks are sufficient. Thus, boundary goals can be seen as the lower limit of a range of possible target goals, complementing previous research that has often focused on individuals' levels of aspiration (e.g., Lewin, 1958; Locke \& Latham, 2002). Levels of aspiration represent the performance standard that individuals are striving to achieve, and these standards may often exceed individuals' boundary goal levels. Indeed, decades of previous research have shown that levels of aspiration positively predict performance, but a major question we investigate is whether individuals' lower boundaries for success also play a role in the achievement striving process.

Consistent with Goal Setting Theory (Locke \& Latham, 1990), we predict that higher target goals should lead to higher performance levels relative to lower target goals. But, in going beyond research on Goal Setting Theory, the current research evaluates whether specific types of target goals also conform to this general principle. Do individuals' lower boundaries for success impact their performance levels, or is it primarily their levels of aspiration that predict success? If it is normative for students to strive for high grades, it may be the case that students' levels of aspirations differ very little between students. Boundary goals, in contrast, are likely to be more variable, and therefore boundary goals may be more predictive of important outcomes than levels of aspiration in such contexts.

A major objective of the current research is to evaluate how target goals are integrated in an overall process model linking higher level achievement goals with performance outcomes by testing associations between achievement goals, target goals (both boundary goals and levels of aspiration), and actual performance in the academic domain. In the achievement goal tradition, performance avoidance goals orient individuals away from negative outcomes whereas performance approach goals orient individuals toward achieving positive outcomes (Elliot \& 
McGregor, 2001). Previous research has shown that performance avoidance goals are associated with worse performance outcomes when compared to performance approach goals, (e.g., Church, Elliot, \& Gable, 2001; Elliot \& Church, 1997; Elliot, McGregor, \& Gable, 1999; Lee, Sheldon, $\&$ Turban, 2003). The current work seeks to explain this association by evaluating a model linking achievement goals to target goals (and particularly boundary goals), which in turn, predict academic performance.

In addition, we also investigate whether target goals, as a sub-goal in the achievement goal hierarchy, can help explain the association between achievement goals and one of their known correlates, study strategies. If target goals are functioning as a sub-goal in this process, they may help explain the connection between achievement goals and study strategies. Thus, we investigate the role of boundary goals (a new type of target goal) and levels of aspiration (a known target goal) in the process of achievement striving. Prior to describing our current model and the present study, we first consider how previous theoretical accounts have conceptualized target goals.

\section{The Function of Target Goals in Achievement-Related Processes}

In classic goal research, target goals were considered an integral part of the goal literature. Kurt Lewin and his students spearheaded a productive line of research in the 1930s and 1940s on level of aspiration, which was defined as the specific level of performance that a person is aiming to achieve (Lewin, Dembo, Festinger, \& Sears, 1944). In the game of darts, for instance, one might express level of aspiration as "aiming for the bull's-eye." Level of aspiration, according to Lewin, serves a motivational function by energizing and directing behavior toward a desired outcome. Given a task to perform, individuals will spontaneously adopt a certain level of performance that they want to "aim for," and this standard is revised dynamically as they gain experience with the task (Lewin et al., 1944). The specific level of aspiration that is chosen depends on several factors including past performance, one's self-efficacy at the task, and knowledge of task performance norms. In general, levels of aspiration increase over time in response to meeting or exceeding previous levels of aspiration (Donovan \& Hafsteinsson, 2006; Lewin, 1958) and decrease in response to failing to meet previous levels of aspiration (see Lewin et al., 1944).

Target goals are also an essential element in Goal Setting Theory (Locke \& Latham, 1990; 2002). Goal Setting Theory is the result of more than 40 years of empirical work comparing difficult and specific goals with vaguer "do your best" goals. Research in this tradition indicates that difficult and specific goals result in better performance than less welldefined goals. This finding holds regardless of whether a goal is set by the individual, an outside agent (e.g., a teacher), or by the individual in collaboration with an outside agent (Latham \& Locke, 2007). Thus, a key insight from this tradition is that a specific and well-defined standard is more psychologically powerful than a less specific goal.

From our perspective, it is also useful to acknowledge the possibility of multiple types of specific target goals. Level of aspiration represents one such target goal, which reflects the goal level at which an individual is aiming. Lewin and his students' classic theorizing, however, revealed a range of possible target goals that individuals could adopt. At the lower end of this range is what we term the lower boundary for success, or boundary goal, which is the minimum performance level that an individual must achieve to subjectively experience success. These boundary goals may be critical to the achievement process for several reasons. First, boundary 
goals may serve to anchor an individual's achievement strivings by marking the baseline for acceptable performance levels. Such an anchor may serve as a strong self-regulatory guide for goal pursuit. Second, boundary goals may be of particular importance for individuals with stronger avoidance orientations because of these individuals' heightened attention to cues that indicate failure at goal striving has occurred. Therefore, by directing individuals' attention toward goal-relevant cues (and away from goal-irrelevant cues) and by motivating goal-directed effort, boundary goals are expected to play a key role in the process of achievement striving.

In addition to the motivational function identified by Lewin, target goals (both boundary goals and levels of aspiration) serve an important self-regulatory function by allowing individuals to track their performance relative to a standard. Two major theories, Social Cognitive Theory (Bandura, 1989) and Cybernetic Control Theory (Carver \& Scheier, 1990) have detailed this regulatory function of target goals. For example, Bandura noted: "Simply adopting a goal, whether an easy or challenging one, without knowing how one is doing, or knowing how one is doing in the absence of a goal, has no lasting motivational impact" (1989, p. 27). This regulatory function was more fully explicated in Cybernetic Control Theory (Carver \& Scheier, 1990, which states that goal pursuit works as a negative feedback (i.e., discrepancy reducing) system in which a person's current state is repeatedly compared to a reference value (that is, a target goal or standard). The detection of a discrepancy results in behaviors aimed at bringing the current state in line with the goal.

\section{Integrating Target Goals with Current Achievement Goals Theories}

In sum, we propose that target goals serve two major functions: one motivational and one regulatory. Motivationally, target goals energize and direct behavior in the service of fulfilling superordinate goals, and they allow individuals to self-regulate by comparing their task performance to a standard. An important remaining question concerns the origins of target goals, a question largely unaddressed to date by Goal Setting Theory and Cybernetic Control Theory. We propose that target goals may serve as sub-goals in a hierarchy of motivation, stemming from broader achievement goal orientations (which are rooted in both early experience and broader temperamental dispositions; Elliot \& Thrash, 2002; Gable, Reis, \& Elliot, 2003). Two related and influential models provide a framework for understanding why this may be the case: Elliot's $2 \times 2$ model of achievement goals (Elliot \& McGregor, 2001), which explains the structure of achievement goals, and the hierarchical model of achievement motivation, which explains their origins and how they are linked to more specific goal-pursuits (Elliot, 2006; Elliot \& Church, 1997).

The $2 \times 2$ achievement goal model specifies two dimensions of achievement goals: valence (approach vs. avoidance) and content (mastery vs. performance). Approach goals orient behavior toward drawing closer to positive (desired) outcomes, whereas avoidance goals orient behavior toward movement away from negative (undesired) outcomes. Mastery goals are concerned with learning and the development of competence, whereas performance goals are concerned with the demonstration of competence. When fully crossed, these two dimensions result in four different types of achievement goals. That is, both mastery and performance goals can be directed toward approaching positive outcomes and avoiding negative outcomes.

The hierarchical model of motivation (Elliot, 2006) asserts that the broad achievement goals specified in the 2 x 2 framework can give rise to more specific sub-goals. These sub-goals specify how one might go about achieving higher-level goals. The notion that goals are 
structured hierarchically - that is, from broader to more specific plans of action - has been a part of many theoretical accounts of goal-directed behavior (e.g., Carver, Sutton, \& Scheier, 2000; Elliot \& Church, 1997; Vallacher \& Wagner, 1987) but has not yet been explored empirically in the context of target goals as a sub-goal in the achievement goal hierarchy.

Using these theoretical ideas, we propose that one key source of target goals may be an individual's higher-level achievement goals. Target goals are a logical candidate for status as a sub-goal in the achievement goal hierarchy because they are more concrete than higher-level achievement goals and because they are contextualized within a given goal pursuit setting. We further posit that a focus on approaching positive outcomes (approach goal orientation) should orient individuals toward achieving higher aims, and therefore, these individuals will set higher levels of aspiration and higher boundaries for success. Further, a focus on avoiding negative outcomes (avoidance goal orientation) should orient individuals toward setting standards at an easily surpassable (i.e., low) level. Boundary goal levels should be particularly affected by this orientation because it is the boundary goal level that indicates when some minimal level of acceptable performance has been achieved. Given that students' levels of aspiration may be more uniformly high than their boundary goals (i.e., many students may say that want to achieve a grade point average of 4.0), we would expect levels of aspiration to be less affected by achievement orientation than boundary goals.

As a sub-goal in the achievement goal hierarchy, target goals should stem from individuals' achievement goals, and they should also have an impact on achievement relevant outcomes. Two achievement relevant outcomes are of interest in the current research: course performance and study strategies. Considering study strategies first, previous research has suggested that different achievement goals lead to different types of study strategies, which are then related to subsequent course performance (Elliot, McGregor, \& Gable, 1999). In particular, mastery approach goals are positively associated with deep processing strategies, persistence, and effort, performance approach goals are positively associated with surface processing strategies, persistence, and effort, and performance avoidance goals are negatively associated with deep processing strategies, but positively predict surface processing and disorganized study strategies. Disorganization is negatively associated with performance, whereas persistence and effort are positively associated with performance. Surface and deep processing typically exhibit small or null associations with performance. Our model predicts that target goals, as a sub-goal in the goal hierarchy, should serve a more proximal role in directing strategy adoption than achievement goals themselves. Given the motivational and regulatory functions of target goals outlined above, we predict that target goals will have the strongest association with strategies reflecting greater goal-directed effort (high effort and persistence) and more focused attention to goal relevant activities (low disorganization). Finally, target goals should mediate the association between achievement goals and study strategies.

Regarding course performance, there is a wealth of evidence suggesting higher levels of aspiration predict better task performance (Lewin, 1958; Locke \& Latham 2002). Because boundary goals and levels of aspiration are conceptualized as different points of a range of possible target goals, they should be highly associated with one another, and both goals should predict course performance. However, if boundary goals exhibit more variability than levels of aspiration, we would expect them to predict course outcomes more strongly than levels of aspiration. In sum, we propose (in agreement with the hierarchical model; see Hulleman, Schrager, Bodmann, \& Harackiewicz, 2010) that broad motives and temperaments provide the energization (the "why") for goal-directed behavior, achievement goals specify the desired 
outcome (the "what"), and more concrete sub-goals (such as target goals) indicate the means (the "how") by which desired outcomes can be achieved (cf. Scholer \& Higgins, 2008).

\section{Summary of Proposed Model}

Combining classical and contemporary perspectives on goals may provide a more complete picture of the role that target goals are expected to play in achievement settings. Specifically, our model predicts that approach achievement goals should be associated with higher target goals because of their focus on positive possibilities, whereas avoidance achievement goals should be associated with lower target goals because of their focus on negative possibilities. It should be noted that this prediction is not simply a tautology - there is nothing inherent to the constructs of approach and avoidance goals that says one should be associated with higher goal levels than the other. Indeed, target goal level and achievement goal content are conceptually independent from one another. For instance, striving to avoid looking incompetent compared to one's classmates (performance avoidance goal content) could be present in individuals with any goal level because 'looking incompetent' is a relative term. Thus, our model predicts differences in target goal level based on achievement goal content, but there is nothing inherently circular about this prediction.

In the current research, we measured target goals in terms of desired performance outcomes. Although we could have also assessed mastery-based target goals (e.g., percent of course content one is striving to learn), it seemed difficult to do so without a concrete masterybased performance criterion. In contrast grades in the course are a very concrete performancebased criterion. Further, it has been noted previously (e.g., Hulleman et al., 2010) that goals about performance levels (e.g., "Get an A on the exam") can stem from mastery-based motivation (e.g., "The purpose of taking this class is to learn all I can") because fulfillment of the performance standard may indeed reflect mastery of the material. Similarly, performanceoriented target goals (i.e., boundary goals and levels of aspiration) might be associated with mastery achievement goal orientation because achieving a specific target level of performance may signal that the overarching mastery goal has also been fulfilled. Thus, in the subsequent analyses, we investigate the possibility that both mastery and performance achievement goals are associated with target goals and that target goals mediate the associations between both types of goals and performance outcomes.

There are at least two main potential contributions of the current research. First, we seek to integrate target goals into the hierarchical model of achievement motivation by specifying a process model that links achievement goals (a higher order goal) to exam performance (an outcome) through target goals (a sub-goal) and study strategies (a means for achieving higher order and sub-goals). Such a model can be viewed an extension of previous work (e.g., Elliot, McGregor, \& Gable, 1999) that identified study strategies as an important part of the process connecting achievement goals to academic performance. Second, we investigate the role of a new type of target goal, boundary goals, as well as an established target goal, level of aspiration, in this process. We predicted that approach goal orientation would be associated with higher target goal levels, whereas avoidance goal orientation would be associated with lower target goal levels - especially lower boundary goal levels. We further predicted that boundary goal levels would predict course performance and study strategies above and beyond levels of aspiration.

\section{Method}




\section{Participants and Procedure}

Participants were recruited from a psychological research methods course that is required of all psychology majors at a large Midwestern research university. The data were collected during two semesters in which the course was taught by same instructor (Fall 2008 and Spring 2009). Students received extra course credit for participating in this study and other research studies (if they so chose). Of 446 students who were eligible to participate, 347 enrolled in the study and consented to participate (78\% participation rate). Participants were mostly juniors and seniors $(90 \%)$, White $(82 \%)$, and female $(76 \%)$. Participants were recruited for the study on the first day of class, at which time they were informed that although some of the data collection would take place during class, their status as a participant or a non-participant would not be made known to the instructor and would have no impact on their grades in the class; students gave additional consent to release their grades to the researchers. The first author, who was not affiliated with the course, collected the data while the instructor was absent.

Overall, there were five occasions of assessment in the study, plus the final grades. After agreeing to participate in the study, participants received a baseline questionnaire packet that they completed at home and returned on the second day of class. The baseline questionnaire packet contained questions about students' personalities and achievement goals, their baseline goals for the course, and demographic questions. In the week prior to the first exam, another assessment was administered in class, assessing study strategies specific to the research methods course. Each of the three exams represented an occasion of measurement; these scores were obtained at the conclusion of the course from course records.

\section{Measures}

Descriptive statistics for each of the measures are displayed in Table 1.

Goal measures. Achievement goals were measured at baseline using the Achievement Goals Questionnaire (Elliot \& McGregor, 2001). This inventory is comprised of 12 items with 3 items per subscale; responses range from 1 (strongly disagree) to 5 (strongly agree). The subscales assess students' mastery approach (e.g., "I want to learn as much as possible from this class"), mastery avoidance (e.g., "I worry that I may not learn all that I possibly could in this class"), performance approach (e.g., "It is important for me to do better than other students"), and performance avoidance (e.g., "My goal in this class is to avoid performing poorly") goals.

Students' target goals were also assessed at baseline using single-item measures designed to tap a range of students' target goals, including lower boundaries for success ("What's the lowest grade in this course that would be acceptable to you?") and target levels of aspiration ("What grade are you aiming for in this course?"). The response scale was in grade point average (GPA) units with response options ranging from 1.0 to 4.0 in half-point increments (consistent with the system of grading used at the university where the data were collected).

Course performance. Student performance in the research methods course was comprised of three exams $(60 \%)$, eight homework exercises $(16 \%)$, and a final course paper $(24 \%)$. For the purposes of the current research, we consider only performance on the three exams. Each multiple-choice exam was worth 100 points. Grades in the course were not normatively determined (i.e., not curved). ${ }^{1}$

As in previous research (e.g., Elliot \& McGregor, 2001), all analyses control for selfreported ACT score (a standardized achievement test required for admission at the university 
where the data were collected) to control for variability in performance that is attributable to academic ability and to address the concern that participants with avoidance goals perform worse in the course simply because they have less academic ability at the study's onset. However, the correlations between primary study variables and self-reported ACT scores were not particularly large (i.e., largest $r=.32$; see Table 2).

Study strategies. Course specific study strategies were assessed using Elliot, McGregor, and Gable's (1999) measure just prior to exam 1. The measure is composed of 21 items assessing deep processing (5 items; e.g., "I try to think through topics and decide what I'm supposed to learn from them, rather than studying topics by just reading them over."), surface processing (5 items; e.g., "When I study for the exam, I try to memorize as many facts as I can."), disorganization (e.g., "I'm not sure how to study for this course."), persistence (e.g., "Regardless of whether or not I like the material, I work my hardest to learn it."), and effort (e.g., "I worked very hard to prepare for the exam.").

An exploratory factor analysis with principal axis factoring and an oblimin rotation suggested that a four-factor structure was more reasonable than the proposed five-factor structure (according to the criteria of simple structure), with the persistence and effort items loading on the same factor and all other items loading on their proposed factors. The bivariate correlation between persistence and effort scales $(r=.68)$ further justified combining these scales. For simplicity, we used the four-factor structure, naming the new combined dimension of effort and persistence - "tenacity." The reliabilities for the scales were acceptable with $\alpha$ 's ranging from .69 (surface processing) to .88 (disorganization; see Table 1).

\section{Data Analysis}

The data were analyzed using the structural equation modeling program Mplus (Version 4.1; Muthén \& Muthén, 2006). There were some limited amounts of missing data dispersed throughout the dataset (e.g., as when a participant skipped an item or missed a wave of data collection). Over $90 \%$ of participants completed each of the waves from which the variables were taken. For the analyses reported here, Mplus handles missing data using a maximum likelihood estimation technique (full imputation maximum likelihood, or FIML; a well recommended method for handling missing data according to Widaman, 2006). Therefore, all analyses are based on information from the full sample size $(n=347)$.

Model fit was considered acceptable if CFI $\geq .95$, RMSEA $\leq .06$, and SRMR $\leq .08$ (Hu \& Bentler, 1999). Although we had no hypotheses concerning the role of participant sex, we controlled for the effect of sex in all analyses by correlating it with all exogenous variables and regressing endogenous variables onto it (sex was dummy-coded, $0=$ female, $1=$ male). We use Cohen's rule of thumb for interpreting the size of all correlation coefficients and standardized regression coefficients (an effect of about $|.10|=$ small, an effect of about $|.30|=$ moderate, and an effect $\geq|.50|=$ large). Regarding mediated effects, we follow Preacher and Kelley (2011) in interpreting a mediated effect of about $|.01|$ as small, about $|.09|$ as moderate, and $\geq|.25|$ as large. Throughout the text we report standardized coefficients (betas) to ease interpretation, but we also include the standard error of the unstandarized coefficients and associated $t$-values (from which the interested reader can calculate the unstandardized coefficients).

In all analyses testing for mediation, we follow the more contemporary recommendations that have been made since the publication of the classic Baron and Kenny (1986) paper (see e.g., Shrout \& Bolger, 2002). In particular, we examine the size and significance of the indirect 
(mediated) effects using bias-corrected, bootstrapped confidence intervals with 5,000 resamples (the recommended method according to MacKinnon, Fairchild, \& Fritz, 2007). This technique allows us to calculate empirically derived standard errors for all model parameters including indirect effects. We then examined the asymmetric 95\% confidence interval derived from the bootstrapping procedure to determine if a given parameter was statistically significant by inspecting whether the $95 \%$ confidence interval included zero. Thus, the $t$-values associated with these parameters may not match the conclusions drawn from the bootstrapped confidence interval because the distribution of these parameters is unlikely to be normal.

\section{Preliminary Analyses}

\section{Results}

Exam performance over time. We first conducted a latent growth curve analysis of students' performance on the three exams with ACT score and sex predicting both the level and the slope factors. To identify the intercept, the loadings for all three exams were fixed to one. To identify the slope, the loading for the first exam was fixed to zero and the loading for the last exam was fixed to one. The loading for the second exam was freely estimated. The mean of the slope parameter therefore represents how much the average individual's exam score changed from the first to the last exam. The model fit the data well, $\chi^{2}(4)=1.79, p=.775, \mathrm{CFI}=1.00$, $\mathrm{RMSEA}=.00, \mathrm{SRMR}=.02$. The loading for the second exam was -1.25 , reflecting the fact that exam scores decreased between the first and second exam but increased from the second to the third exam. The mean of the level $(M=76.01, S E=.81, t=93.53)$ and the slope $(M=1.85, S E=$ $.82, t=2.25)$ of exam scores were both statistically significant. Thus, the average individual earned a $76 \%$ on the first exam, about $74 \%$ on the second exam, and $78 \%$ on the third exam. There was significant variability in individuals' performance levels, $S D=9.17, S E=8.54, t=$ 9.85, but there was no variability in individuals' slopes, $S D=.75, S E=6.09, t=.093$. ACT score predicted individuals' mean level of performance, $\beta=.29, S E=.20, t=4.33$, but sex did not, $\beta=.03, S E=1.39, t=0.47$. Neither ACT score nor sex predicted the slope of performance. In the absence of variability in the slope parameter, a no-change (intercept-only) model is perhaps more sensible for the current analyses.

As an initial examination of the effects of target goals on exam performance, we plotted the average observed trajectory of exam scores for individuals with different boundary goal levels (top panel, Figure 1) and levels of aspiration (bottom panel, Figure 1). The marked negative skew in levels of aspiration is worth noting. Only 6 students adopted a level of aspiration less than 3.0, and the majority of students (71\% of respondents) stated that they aspired to achieve a 4.0 in the course. Boundary goals, in contrast, exhibited a more normal distribution with many students adopting different boundary goals. The figures also highlight the fact that average exam scores remain relatively constant over time. Importantly, the top panel of Figure 1 shows a clear delineation in performance levels among students who adopted different boundary goals. To some extent, the bottom panel of Figure 1 shows a similar delineation, however it appears that only the individuals with levels of aspiration equal to 4.0 are differentiable from students with other levels of aspiration.

Associations between key study variables. We conducted a confirmatory factor analysis (CFA) with all latent factors (performance approach goals, performance avoidance goals, mastery approach goals, mastery avoidance goals, surface processing, deep processing, disorganization, tenacity, and exam scores) and all single-item observed variables (ACT score, 
boundary goal, level of aspiration at all time points, and sex). Each latent variable was identified by fixing the loading of the first item to one. A correlation matrix with all variables included in the analysis is displayed in Table 2, and CFA loadings are displayed in Table 3. The model had an acceptable fit to the data, $\chi^{2}(666)=1177.17, p<.001, \mathrm{CFI}=.90, \mathrm{RMSEA}=.05, \mathrm{SRMR}=$ .06 .

The correlation matrix reveals that boundary goals and baseline levels of aspiration were strongly associated with each other, $r=.59, t=9.20, p<.001$, consistent with the hypothesis that both are types of target goals. Further, both types of target goals were associated with the latent exam performance variable. Boundary goals were strongly related to exam performance, $r$ $=.51, t=7.27, p<.001$, whereas levels of aspiration were moderately related to exam performance, $r=.35, t=5.20, p<.001$. Thus, consistent with predictions, higher boundary goals and higher levels of aspiration were associated with better exam performance. This result provided preliminary support for hypotheses about the relevance of boundary goals for achievement outcomes.

Consistent with prior research, performance approach goals were positively associated with exam performance, $r=.25, t=3.80, p<.001$, and performance avoidance goals were negatively associated with exam performance, $r=-.18, t=-2.54, p=.01$. Mastery approach goals were also positively associated with exam performance, $r=.16, t=2.30, p=.02$; mastery avoidance goals were not, $r=-.01, t=-.11, p>.05$. Also consistent with prior research, disorganized study strategies had a negative association with exam performance, $r=-.41, t=$ $4.80, p<.001$. Tenacity was positively associated with exam performance, $r=.33, t=4.37, p<$ .001 . Deep processing had a small positive association with exam performance, $r=.15, t=2.01$, $p=.04$. Surface processing was not associated with exam performance, $r=.05, t=.59, p>.05$. In short, an initial examination of the pattern of observed correlations was consistent with our underlying theoretical model.

\section{Do Target Goals and Study Strategies Mediate the Association between Achievement Goals and Exam Performance?}

The outline of the basic model that we used to test the main study hypotheses is depicted in Figure 2, and parameter estimates associated with that model are reported in Table 4. This model allows for multiple mediational pathways from achievement goals to performance outcomes. First, it specifies a mediational chain from achievement goals to target goals to study strategies and finally to exam performance. Further, it specifies a mediational pathway through target goals that is independent of study strategies, as well as mediation through study strategies that is independent of target goals. The direct effects from achievement goals to performance were also included in this model (to allow for the possibility that the pathways in the model may only partially mediate the effects), and the disturbances of the mediators were allowed to correlate with one another (i.e., one correlation between boundary goals and levels of aspiration and six correlations among the study strategies). ACT score and sex were regressed onto all endogenous variables and correlated with all exogenous variables. The overall model had an acceptable fit to the data, $\chi^{2}(666)=1177.17, p<.001, \mathrm{CFI}=.90, \mathrm{RMSEA}=.05, \mathrm{SRMR}=.06$.

Our first objective was to determine the extent to which each achievement goal was uniquely associated with the target goals. Consistent with predictions, performance approach goals were positively associated with boundary goals $(\beta=.32, S E=.03, t=5.75)$, whereas there was no evidence of a unique effect for performance avoidance goals $(\beta=-.11, S E=.05, t=$ - 
$1.52)^{2}$. Further, mastery approach goals were positively associated with boundary goals ( $\beta=.29$, $S E=.08, t=3.98)$, and mastery avoidance goals were negatively associated with boundary goals $(\beta=-.25, S E=.05, t=-3.42)$. Performance approach goals were positively associated with levels of aspiration $(\beta=.21, S E=.02, t=3.89)$, as were mastery approach goals $(\beta=.35, S E=.07, t=$ 4.19). Mastery avoidance goals were negatively associated with levels of aspiration $(\beta=-.18, S E$ $=.03, t=-2.63)$, and performance avoidance goals were not independently related to levels of aspiration $(\beta=-.08, S E=.04, t=-1.02)$. Further, boundary goals independently were related to exam performance $(\beta=.32, S E=1.65, t=3.92)$, whereas this was not the case for levels of aspiration $(\beta=.09, S E=2.39, t=1.05)$. Thus, consistent with predictions, performance approach goals were uniquely associated with target goals, as were mastery approach and mastery avoidance goals. Performance avoidance goals were not uniquely associated with target goals.

Cumulative mediated effects, direct effects, and total effects. To test our main hypothesis of whether target goals mediate the association between achievement goals and exam performance, we conducted a series of statistical tests. First, we evaluated the size and significance of the cumulative mediated effects (i.e., the total indirect effect) through all proposed mediational pathways, as well as total effects (i.e., the sum of the total indirect effect and the direct effect from each of the achievement goals to performance). The cumulative mediated effect indicates the extent to which target goals and study strategies are collectively serving as mediators of the association between achievement goals and exam performance. The direct effect reflects the extent to which achievement goals are associated with performance after all proposed mediated pathways have been taken into account. The total effect for a given achievement goal is then the summation of all indirect effects and the direct effect associated with that goal.

Considering first the total indirect effects, three of the four achievement goals had significant total indirect effects through the proposed mediators: $\beta=.15, S E=.54, t=2.82$ for performance approach goals, $\beta=.35, S E=1.96, t=3.75$ for mastery approach goals, and $\beta=$ $.26, S E=.89, t=-3.74$ for mastery avoidance goals. The total indirect effect through performance avoidance goals was not statistically significant, $\beta=-.09, S E=1.45, t=-.93$. Thus, the cumulative mediational effect through target goals and study strategies was significant for three of the four achievement goals in the $2 \times 2$ framework.

Regarding direct effects, neither performance approach nor performance avoidance goals had a significant effect on exam performance once the mediators were included in the model $(\beta=$ $.08, S E=.79, t=1.71$ for performance approach and $\beta=-.10, S E=1.84, t=-.76$ for performance avoidance). However, both mastery approach and mastery avoidance goals had significant direct effects, $\beta=-.25, S E=2.36, t=-2.25$ and $\beta=.25, S E=1.12, t=2.81$, respectively. For both goals, these direct effects had a sign opposite to the sign of the indirect effects, a condition that suggests statistical suppression. Given the large number of variables in the model, it is difficult to give a definitive interpretation to these results. Recall that the direct effect accounts for the variance shared between these goals and performance while accounting for the mediated effect. Given that the mediated effect appears to be quite sizable, it is unclear how to precisely interpret this direct effect.

By summing the direct and indirect effects, we found that the total effects were significant and moderate in size for both types of performance achievement goals (total effects $\beta$ $=.24, S E=.70, t=3.51$ for performance approach and $\beta=-.19, S E=1.31, t=-2.10$ for performance avoidance), indicating that the cumulative association between performance approach goals and exam scores was positive and the cumulative effect of performance 
avoidance goals on exam scores was negative. However for the mastery goals, the total effect was not significant because of the opposing signs of the direct and indirect effects (total effects $\beta$ $=.10, S E=1.73, t=1.18$ for mastery approach and $\beta=-.01, S E=.97, t=-.17$ for mastery avoidance). It is also useful to note that the total effects for the mastery goals were consistent with the zero-order correlations reported above.

Specific mediated effects. We then evaluated the indirect effects for specific mediators, focusing on how much of the effects of achievement goals were mediated through each type of target goal, both independent of each other, independent of study strategies, and together with study strategies. These indirect effects indicate how much of a statistical association between achievement goals and performance could be traced through specific mediators in the model, independent of other pathways and the direct effect. In other words, they represent the unique mediational effect of a given variable.

Considering mediated effects through target goals that were independent of study strategies, the model indicates that boundary for success mediates the association between performance approach goals and exam performance $(\beta=.10, S E=.32, t=3.37)$, consistent with predictions. The indirect effect for performance avoidance goals through boundary goals was not significant $(\beta=-.04, S E=.36, t=-1.44)$. Further, boundary for success mediated the association between mastery achievement goals and exam performance $(\beta=.09, S E=.72, t=2.70$ for mastery approach goals; $\beta=-.08, S E=.43, t=2.33$ for mastery avoidance goals). There were no significant indirect effects through level of aspiration that were independent of boundary goals and study strategies (all $t \mathrm{~s}<|1|$ ). These effects suggest that boundary goals account for a sizable independent chunk of the cumulative indirect effect for three of the four achievement goals.

There were also a few significant indirect effects for achievement goals that could be traced through target goals and then study strategies to performance (i.e., via the full mediated path depicted in Figure 2). For performance and mastery approach goals there were small negative indirect effects through level of aspiration, then disorganization $(\beta=-.01, S E=.07, t=$ 1.57 and $\beta=-.02, S E=.24, t=-1.45$, respectively). For both of these goals, there was also a small positive indirect effect through boundary goals, then disorganization $(\beta=.02, S E=.10, t=$ 1.71 and $\beta=.02, S E=.18, t=1.77$ respectively). For mastery avoidance goals, there was a small positive indirect effect through level of aspiration, then disorganization, $\beta=.01, S E=.08, t=$ 1.34, and there was a small negative indirect effect through boundary goals, then disorganization, $\beta=-.01, S E=.09, t=-1.75$. Although small in absolute magnitude, these effects represent the product of three regression coefficients and should therefore be interpreted with care.

Descriptively they represent a small portion of the cumulative indirect effect for three of the four achievement goals.

Last, there were also a handful of significant indirect effects from achievement goals through study strategies to exam performance that were independent of target goals. For mastery approach goals, there were positive effects through tenacity and disorganization $(\beta=.18, S E=$ $2.24, t=1.71$ and $\beta=.08, S E=.80, t=2.21$, respectively). For mastery avoidance goals, there was a negative effect through disorganization, $\beta=-.10, S E=.51, t=-2.50$. These pathways represent associations between the relevant achievement goals and performance that are not mediated by target goals. However, these effects are relatively smaller than the bivariate correlations between these achievement goals and study strategies. It can therefore be concluded that target goals are partially mediating the effect of achievement goals on study strategies.

The final model depicted in Figure 2 and reported in Table 4 accounted for $46.1 \%$ of the variance in exam scores, $35 \%$ of the variance in surface processing, $28 \%$ of the variance in deep 
processing, $39 \%$ of the variance in tenacity, $23 \%$ of the variance in disorganization, $24 \%$ of the variance in level of aspiration, and 33\% of the variance in boundary goals. Moreover, boundary goals accounted for a significant and moderately sized portion of the total mediated effect between achievement goals (viz., performance approach, mastery approach, and mastery avoidance goals) and exam performance. This effect was independent of the effects of level of aspiration, as well as the effect of study strategies. Thus, we found support for our predictions regarding the importance of boundary goals for explaining the observed associations between achievement goals and course performance.

Follow-up analyses. We also evaluated a set of follow-up analyses in which only one target goal at a time was included in the model. For simplicity, we excluded study strategies from this model. These models allow us to avoid the "perils of partialling" (Lynam, Hoyle, \& Newman, 2006) by evaluating how each target goal mediated the effect of achievement goals on exam scores on its own. These models had an acceptable fit to the data, $\chi^{2}(110)=229.66, p<$ $.001, \mathrm{CFI}=.94, \mathrm{RMSEA}=.06, \mathrm{SRMR}=.05$ for boundary goals and $\chi^{2}(110)=241.58, p<$ $.001, \mathrm{CFI}=.93, \mathrm{RMSEA}=.06, \mathrm{SRMR}=.05$ for level of aspiration. Results suggested that each target goal mediated the effect of the achievement goals (performance approach, mastery approach, and mastery avoidance) on exam performance though the effects appeared larger for boundary goals than levels of aspiration. Boundary goals predicted exam performance, $\beta=.43$, $S E=1.46, t=5.73$, as did level of aspiration, $\beta=.31, S E=1.66, t=4.94$.

The indirect effects for performance approach, mastery approach, and mastery avoidance goals on exam performance through boundary goals $(\beta \mathrm{s}=.14, .12$, and -.11 , respectively $)$ and levels of aspiration $(\beta \mathrm{s}=.05, .08$, and -.04$)$ were significant. As in the analysis above, neither boundary goals nor levels of aspiration mediated the effect of performance avoidance goals on exam performance $(\beta \mathrm{s}=-.05,-.02)$. Taken together the results suggest that level of aspiration, although predictive of performance on its own, does not predict performance above and beyond the effects of boundary goals, lending support to our initial claim that boundary goals may be a particularly useful goal for educators.

\section{Discussion}

The objective of the current manuscript was to more fully integrate target goals into Elliot's (2006) hierarchical model of achievement motivation. We hypothesized that boundary goals (a new type of target goal) would relate to academic performance above and beyond level of aspiration (a better known target goal) and that boundary goals would mediate the associations between achievement goals and performance. At a general level, we found support for these hypotheses.

Consistent with expectations, we found evidence that boundary goals are better predictors of performance than levels of aspiration when these goals are pitted against one another. Both types of target goals were positively associated with exam performance, but only boundary goals were uniquely associated with performance when both were used as simultaneous predictors. We believe this result is driven by two factors: 1) many students seem to aspire to achieve high grades but; 2) some students will settle for lower grades (i.e., they have low boundaries for success), whereas others will not (they have high boundaries for success). Indeed, this would seem to be the real substance of our contribution: the idea that boundary goals might be a more psychologically powerful predictor of effort and performance than levels of aspiration (at least in the kinds of classroom settings common to many universities). 
Beyond providing evidence for a potentially important role for boundary goals in classroom settings, we attempted to embed target goals (both boundary goals and levels of aspiration) within the hierarchical model of achievement motivation (Elliot, 2006). We found that boundary goals mediated the association between performance approach, mastery approach, and mastery avoidance achievement goals and exam performance in a model that included level of aspiration. In contrast, level of aspiration did not mediate these effects when controlling for boundary goals. At a bivariate level, performance avoidance goals were negatively associated with target goals and subsequent performance, but performance avoidance goals did not uniquely predict target goals and target goals did not mediate effects on performance when the other three types of achievement goals and sex were considered, so this particular hypothesis was not supported.

Our final major hypothesis was that as a sub-goal in the achievement goal hierarchy, target goals should influence strategies for goal directed behavior. The actual results involving study strategies were quite complex. We found that a portion of the indirect effect between achievement goals and exam performance was mediated through target goals and then study strategies (specifically disorganization). There were also some effects that were mediated through study strategies (tenacity and disorganization) that were independent of target goals, reflecting the fact that only part of the effect from achievement goals to study strategies was mediated by target goals. It should also be noted that although target goals partially mediated the association between performance approach, mastery approach, and mastery avoidance goals and disorganized study strategies, the individual indirect effects through boundary goals and levels of aspiration were signed in opposite directions. Boundary goals were negatively associated with disorganization, whereas level of aspiration was positively associated with it. It is difficult to interpret this result as the beta coefficient for the effect of level of aspiration on disorganization had the opposite sign from its zero-order correlation. The results regarding boundary goals, however, were consistent with hypotheses.

Considering the results involving study strategies as a whole, then, we found mixed support for our hypotheses. Overall the sizes of the indirect effects were relatively small, though many were statistically significant when using bootstrapping methods for significance testing. Although we measured study strategies specific to the course, they may have been assessed more broadly than they ought to have been. Perhaps a better tactic would have been to measure strategies at the more contextualized level of the individual exam, but for now this question will have to be investigated in future research.

The current results have a number of theoretical implications. First, they provide some support for our hypothesis that target goals (especially boundary goals) function as a sub-goal in the achievement goal hierarchy. Thus, the current work helps to further identify some of the potential mechanisms that may help explain why the goals in the $2 \times 2$ framework are ultimately associated with performance outcomes. Second, our results extend the basic principles of goal setting theory. Considerable research has found support for goal setting theory's basic prediction that specific, difficult goals (i.e., levels of aspiration) lead to higher levels of performance than easier goals. Our results confirm this prediction and extend this idea to boundary goals. By highlighting a distinction between levels of aspiration and boundary goals, this work emphasizes the potential importance of differentiating among different kinds of target goals. The finding that boundary goals outperform levels of aspiration in terms of predictive ability when the two are pitted against one another suggests that goal setting theory researchers should consider making distinctions between different kinds of target goals beyond levels of aspiration. This 
recommendation might be especially relevant when there is a normative press for a certain kind of aspiration, as seems to be in typical college settings.

The results may also have implications regarding the functions of target goals outlined by cybernetic and social cognitive theories. One possibility is that boundary goals may serve as even more potent regulators of behavior than levels of aspiration. This makes sense if boundary goals are seen to represent the dividing line differentiating successful from unsuccessful performance. As such, they represent "a bottom line" standard that can be used to compare goals to actual performance, which could have consequences for determining future behavioral and affective responses. Thus, boundary goals might be the reference value used in cybernetic theories of motivation. By contrast, levels of aspiration seem to reflect more idealized and even wishful standards as compared to boundary goals. Therefore, we suggest that boundary goals are likely to be more consequential for self-regulation.

Although the analyses provide support for many of our hypotheses, there are several limitations concerning the data and analyses worth noting. First, these data are correlational, which constrains the causal inferences that can be drawn from the analyses. We should note, however, that students provided reports of initial target goals on the first day of class, well before the first exam. Thus, the design of this study provides some clarity with respect to temporal precedence when considering the associations between target goals and exam performance. Second, this study investigated target goals in the context of college students' academic performance, and the results may not generalize to non-college student populations or other achievement contexts. In the workplace, for instance, uniformly high levels of aspiration may not be as normative as they appear to be in academic settings. Thus, levels of aspiration may be more informative in other achievement contexts than they were in the current study. Likewise, it remains to be seen whether boundary goals would be as predictive of performance in the workplace as they were in the classroom. Third, the data in the present analysis were pooled from the same course taught by the same instructor, but from two different semesters. Although the analysis would have been underpowered had we examined these sources of data separately, caution is nonetheless warranted in interpreting our results as pooling may result in underestimated standard errors and biased parameter estimates. Finally, since our study was conducted the achievement goal questionnaire has been revised (Elliot \& Murayama, 2008); our analyses used the older version the scale.

Despite these limitations, we believe that the results of the current study are notable for their consistency with theoretically-derived predictions. They are a significant first step towards documenting the importance of boundary goals in achievement motivation processes. These results also have rather straightforward applied implications for promoting achievement. Quite simply, it might be worthwhile to acknowledge distinctions between level of aspiration and boundary goals and attempt to boost boundary goals directly. Indeed, our data show that many students aspire to achieve high grades in their courses, but they seem to vary in their willingness to tolerate lower grades. Imagine the difference between the student who says, "I won't accept anything less than a letter grade of B" (boundary goal = 3.0) and the student who says, "I'll be satisfied if I just pass this class" (boundary goal =1.0). Our data suggest that the former student will perform better in the course than the latter. Perhaps by encouraging students to set higher boundary goals for themselves at the start of a semester, we could help launch students on a trajectory toward higher performance levels. This insight brings us full circle to the opening quotation - a real danger in goal pursuit is not in aiming too high and failing to achieve such a mark, but in attaining a boundary goal that was set too low in the first place. 


\section{References}

Bandura, A. (1989). Self-regulation of motivation and action through internal standards and goal systems. In L. A. Pervin (Ed.), Goal concepts in personality and social psychology (pp. 19-85). Hillsdale, NJ: Lawrence, Erlbaum, Associates, Inc.

Carver, C. S., \& Scheier, M. F. (1990). Origins and functions of positive and negative affect: A control-process view. Psychological Review, 97, 19-35.

Carver, C. S., Sutton, S. K., \& Scheier, M. F. (2000). Action, emotion, and personality: Emerging conceptual integration. Personality and Social Psychology Bulletin, 26, 741751.

Church, M. A., Elliot, A. J., \& Gable, S. L. (2001). Perceptions of classroom environment, achievement goals, and achievement outcomes. Journal of Educational Psychology, 93, 43-54.

Donovan, J. J., \& Hafsteinsson, L. G. (2006). The impact of goal-performance discrepancies, self-efficacy, and goal orientation on upward goal revision. Journal of Applied Social Psychology, 36, 1046-1069.

Elliot, A. J. (2006). The hierarchical model of approach and avoidance motivation. Motivation and Emotion, 30, 111-116.

Elliot, A. J., \& Church, M. A. (1997). A hierarchical model of approach and achievement motivation. Journal of Personality and Social Psychology, 72, 218-232.

Elliot, A. J., \& McGregor, H. A. (2001). A 2 X 2 achievement goal framework. Journal of Personality and Social Psychology, 80, 501-519.

Elliot, A. J., McGregor, H. A., \& Gable, S. L. (1999). Achievement goals, study strategies, and exam performance: A mediational analysis. Journal of Educational Psychology, 91, 549563.

Elliot, A. J., \& Thrash, T. M. (2002). Approach-avoidance motivation in personality: Approach and avoidance temperaments and goals. Journal of Personality and Social Psychology, 82, 804-818.

Elliot, A. J., \& Murayama, K. (2008). On the measurement of achievement goals: Critique, illustration, and application. Journal of Educational Psychology, 100, 613-628.

Gable, S. L., Reis, H. T., \& Elliot, A. J. (2003). Evidence for bivariate systems: An empirical test of appetition and aversion across domains. Journal of Research in Personality, 37, 349372.

Harackiewicz, J. M., \& Sansone, C. (1991). Goals and intrinsic motivation: You can get there from here. In M. L. Maehr \& P. R. Pintrich (Eds.), Advances in motivation and achievement (Vol. 7, pp. 21-49). Greenwich, CT: JAI Press.

Hu, L. \& Bentler, P. M. Cutoff criteria for fit indexes in covariance structure analysis: Conventional criteria versus new alternatives. Structural Equation Modeling, 6, 1-55.

Hulleman, C. S., Schrager, S. M., Bodmann, S. M., and Harackiewicz, J. M. (2010). A metaanalytic review of achievement goal measures: Different labels for the same construct or different constructs with similar labels? Psychological Bulletin, 136, 422-449.

Latham, G. P., \& Locke, E. A. (2007). New developments in and directions for goal-setting research. European Psychologist, 12, 290-300.

Lee, F. K., Sheldon, K. M., \& Turban, D. B. (2003). Personality and the goal-striving process: The influence of achievement goal patterns, goal level, and mental focus on performance and enjoyment. Journal of Applied Psychology, 88, 256-265. 
Lewin, K. (1958). Psychology of success and failure. In C. L. Stacey \& M. F. DeMartino (Eds.), Understanding human motivation (pp. 317-344). Cleveland: Howard Allen, Inc. Publishers.

Lewin, K., Dembo, T., Festinger, L., \& Sears, P. S. (1944). Level of aspiration. In J. M. Hunt (Ed.), Personality and the behavior disorders (pp.333-378). New York: Ronald Press.

Locke, E. A., \& Latham, G. P. (1990). A theory of goal setting and task performance. Englewood Cliffs, NJ: Prentice Hall.

Locke, E. A., \& Latham, G. P. (2002). Building a practically useful theory of goal setting and task motivation. American Psychologist, 57, 705-717.

Lynam, D. R., Hoyle, R. H., \& Newman, J. P. (2006). The perils of partialling: Cautionary tales from aggression and psychopathy. Assessment, 13, 328-341.

MacKinnon, D. P., Fairchild, A. J., \& Fritz, M. S. (2007). Mediation analysis. Annual Review in Psychology, 58, 593-614.

Muthén, L.K. and Muthén, B.O. (1998-2007). Mplus User's Guide. Fifth Edition. Los Angeles, CA: Muthén \& Muthén.

Preacher, K. J., \& Kelley, K. (2011). Effect size measures for mediation models: Quantitative strategies for communicating indirect effects. Psychological Methods, 16, 93-115.

Scholer, A. A., \& Higgins, E. T. (2008). Distinguishing levels of approach and avoidance: An analysis using regulatory focus theory. In A. J. Elliot (Ed.), Handbook of Approach and Avoidance Motivation (pp. 489-504). New York: Psychology Press.

Shrout, P. E., \& Bolger, N. (2002). Mediation in experimental and non-experimental studies: New procedures and recommendations. Psychological Methods, 7, 422-445.

Vallacher, R. R., and Wegner, D. M. (1987). What do people think they're doing? Action identification and human behavior. Psychological Review, 94, 3-15.

Widaman, K. F. (2006). Missing data: What to do with or without them. Monographs of the Society for Research in Child Development, 71, 42-64.

\section{Footnotes}

${ }^{1}$ Exam scores were selected as the dependent variable because this component of the course had the most variability and because there were 3 independent measurement occasions). Students also completed a final paper and lab exercises but scores on these elements of the final grade were more restricted. Exam scores were correlated $r=.79$ with total course points. Furthermore, the current results generally converge with prior research that has used normatively referenced grading scales. Our interpretation of this convergence is that students socially compare their grades regardless of whether or not the class is graded on a curve.

${ }^{2}$ This coefficient is statistically significant in analyses excluding mastery goals and in analyses excluding sex, suggesting that there is overlap in the variance accounted for in target goals by performance avoidance goals and other study variables. 
Table 1

Descriptive Statistics

\begin{tabular}{|c|c|c|c|c|c|c|c|}
\hline & $n$ & $M$ & $S D$ & $\alpha$ & $\min$ & $\max$ & $\begin{array}{c}\text { possible } \\
\text { range }\end{array}$ \\
\hline 1. Level of Aspiration & 315 & 3.78 & 0.38 & - & 2.00 & 4.00 & $1.0-4.0$ \\
\hline 2. Boundary Goal & 319 & 2.96 & 0.51 & - & 1.00 & 4.00 & $1.0-4.0$ \\
\hline 3. Performance Approach & 347 & 3.18 & 1.07 & 0.88 & 1.00 & 5.00 & $1-5$ \\
\hline 4. Performance Avoid & 347 & 3.77 & 0.89 & 0.72 & 1.00 & 5.00 & $1-5$ \\
\hline 5. Mastery Approach & 347 & 3.83 & 0.74 & 0.78 & 1.67 & 5.00 & $1-5$ \\
\hline 6. Mastery Avoid & 347 & 3.00 & 0.87 & 0.78 & 1.00 & 5.00 & $1-5$ \\
\hline 7. Exams & 332 & 229.00 & 31.85 & 0.79 & 135.00 & 300.00 & $0-300$ \\
\hline 8. Total Points & 344 & 408.00 & 74.23 & - & 16.50 & 517.50 & $0-520$ \\
\hline 9. ACT Score & 300 & 24.82 & 3.25 & - & 13 & 32 & $1-36$ \\
\hline 10. Disorganization & 308 & 2.55 & 0.94 & 0.88 & 1.00 & 5.00 & $1-5$ \\
\hline 11. Surface Processing & 308 & 3.59 & 0.67 & 0.69 & 1.80 & 5.00 & $1-5$ \\
\hline 12. Deep Processing & 308 & 2.89 & 0.72 & 0.74 & 1.20 & 4.80 & $1-5$ \\
\hline 13. Tenacity & 308 & 3.52 & 0.72 & 0.86 & 1.67 & 5.00 & $1-5$ \\
\hline
\end{tabular}




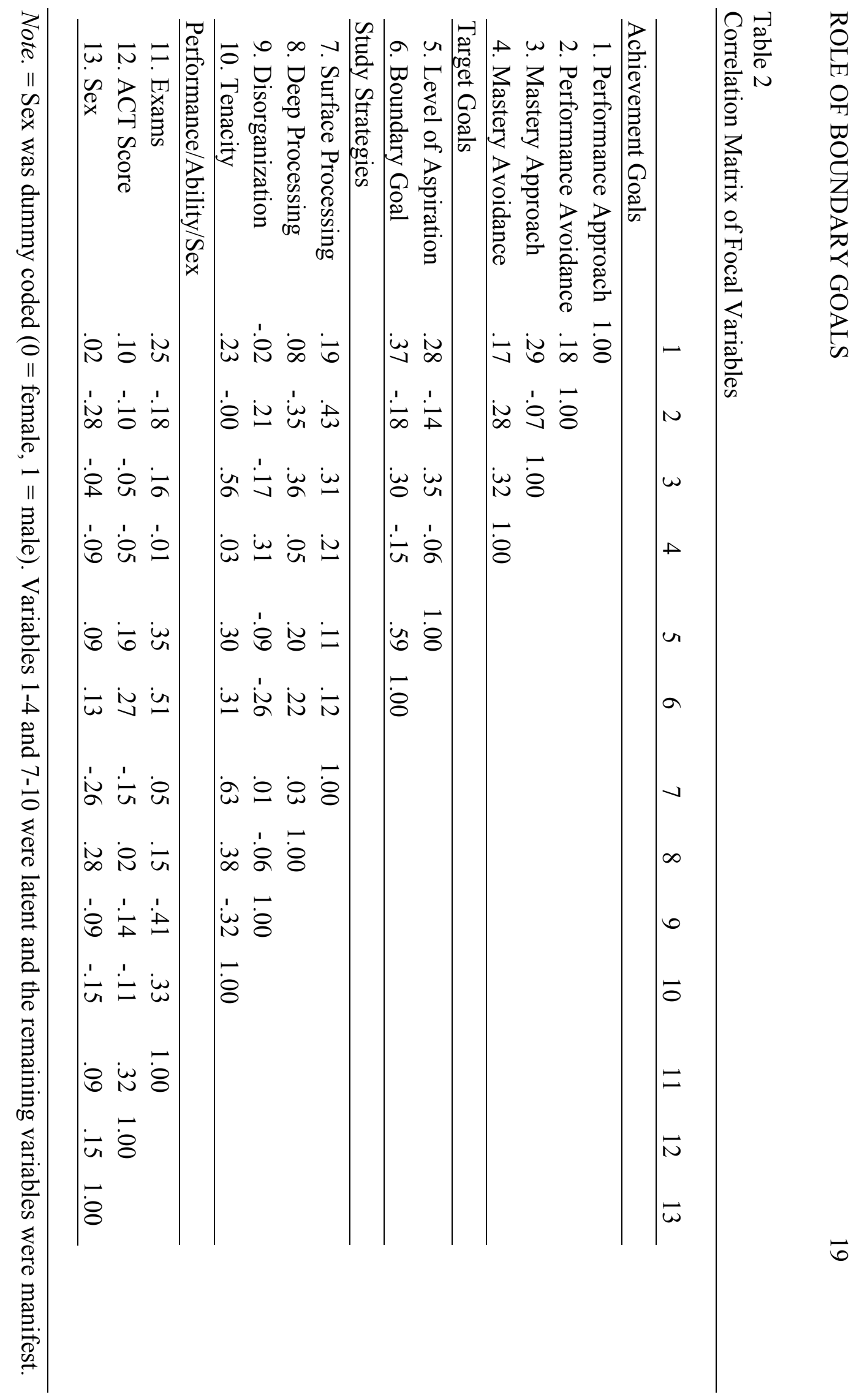


Table 3

Confirmatory Factor Analysis Loadings

\begin{tabular}{|c|c|c|c|c|}
\hline Scale & Abbreviated Item Text & $b$ & $S E$ & $\beta$ \\
\hline \multirow{3}{*}{$\begin{array}{c}\text { Performance } \\
\text { Approach }\end{array}$} & Goal is to get a better grade than most others & 1.00 & - & .85 \\
\hline & Important for me to do better than other students & 1.07 & 0.06 & .89 \\
\hline & Important for me to do well compared to others & 0.93 & 0.06 & .80 \\
\hline \multirow{3}{*}{$\begin{array}{c}\text { Performance } \\
\text { Avoidance }\end{array}$} & Goal is to avoid performing poorly & 1.00 & 0.00 & .74 \\
\hline & Fear of performing poorly motivates me & 0.99 & 0.11 & .59 \\
\hline & Just want to avoid doing poorly & 1.17 & 0.13 & .73 \\
\hline \multirow{3}{*}{$\begin{array}{l}\text { Mastery } \\
\text { Approach }\end{array}$} & Want to learn as much as possible & 1.00 & - & .61 \\
\hline & Important to understand content thoroughly & 1.36 & 0.13 & .78 \\
\hline & Desire to completely master the material & 1.67 & 0.16 & .82 \\
\hline \multirow{3}{*}{$\begin{array}{c}\text { Mastery } \\
\text { Avoidance }\end{array}$} & Concerned I may not learn all there is to learn & 1.00 & - & .78 \\
\hline & Afraid I may not understand content as thoroughly as I should & 0.74 & 0.07 & .60 \\
\hline & Worry I may not learn all that I possibly could & 1.07 & 0.08 & .86 \\
\hline \multirow{5}{*}{$\begin{array}{c}\text { Surface } \\
\text { Processing }\end{array}$} & Try to memorize everything I think will be on the exam & 1.00 & - & .55 \\
\hline & Study by memorizing the chapter definitions in the text & 1.04 & 0.16 & .49 \\
\hline & Read the text and my notes over and over & 1.30 & 0.18 & .69 \\
\hline & Find the most important ideas in text/notes to memorize & 0.61 & 0.11 & .41 \\
\hline & Try to memorize as many facts as I can & 1.10 & 0.14 & .62 \\
\hline \multirow{5}{*}{$\begin{array}{c}\text { Deep } \\
\text { Processing }\end{array}$} & Think through topics and decide what I'm supposed to learn & 1.00 & - & .45 \\
\hline & Try to decide if there is good supporting evidence for theory & 1.83 & 0.27 & .75 \\
\hline & Develop my own ideas about course material & 1.84 & 0.26 & .78 \\
\hline & Never question the validity of the theories presented (R) & 1.01 & 0.21 & .38 \\
\hline & Think about possible theoretical alternatives & 1.52 & 0.23 & .68 \\
\hline \multirow{5}{*}{ Disorganization } & Find it difficult to organized my study time effectively & 1.00 & - & .53 \\
\hline & Have trouble figuring out what to do to learn the material & 1.36 & 0.15 & .77 \\
\hline & Not sure how to study for this course & 1.66 & 0.17 & .89 \\
\hline & Find it difficult to develop a study plan & 1.60 & 0.16 & .88 \\
\hline & Don't know what to study or where to start & 1.56 & 0.16 & .82 \\
\hline \multirow{6}{*}{ Tenacity } & Spend extra time and effort understanding difficult topics & 1.00 & - & .72 \\
\hline & When confused with reading, go back and try to figure it out & 1.07 & 0.08 & .78 \\
\hline & Learn all testable material "inside and out" even if it is boring & 0.78 & 0.08 & .65 \\
\hline & Regardless of whether I like it, I work my hardest to learn it & 0.64 & 0.07 & .59 \\
\hline & Put a lot of effort into preparing for the exam & 1.01 & 0.09 & .75 \\
\hline & Worked very hard to prepare for the exam & 1.11 & 0.09 & .81 \\
\hline \multirow{3}{*}{ Exams } & 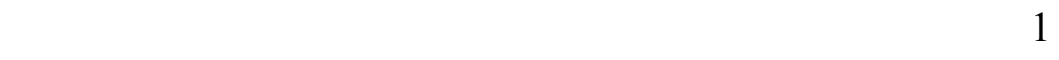 & 1.00 & - & .80 \\
\hline & 2 & 0.89 & 0.08 & .73 \\
\hline & 3 & 0.86 & 0.08 & .70 \\
\hline
\end{tabular}




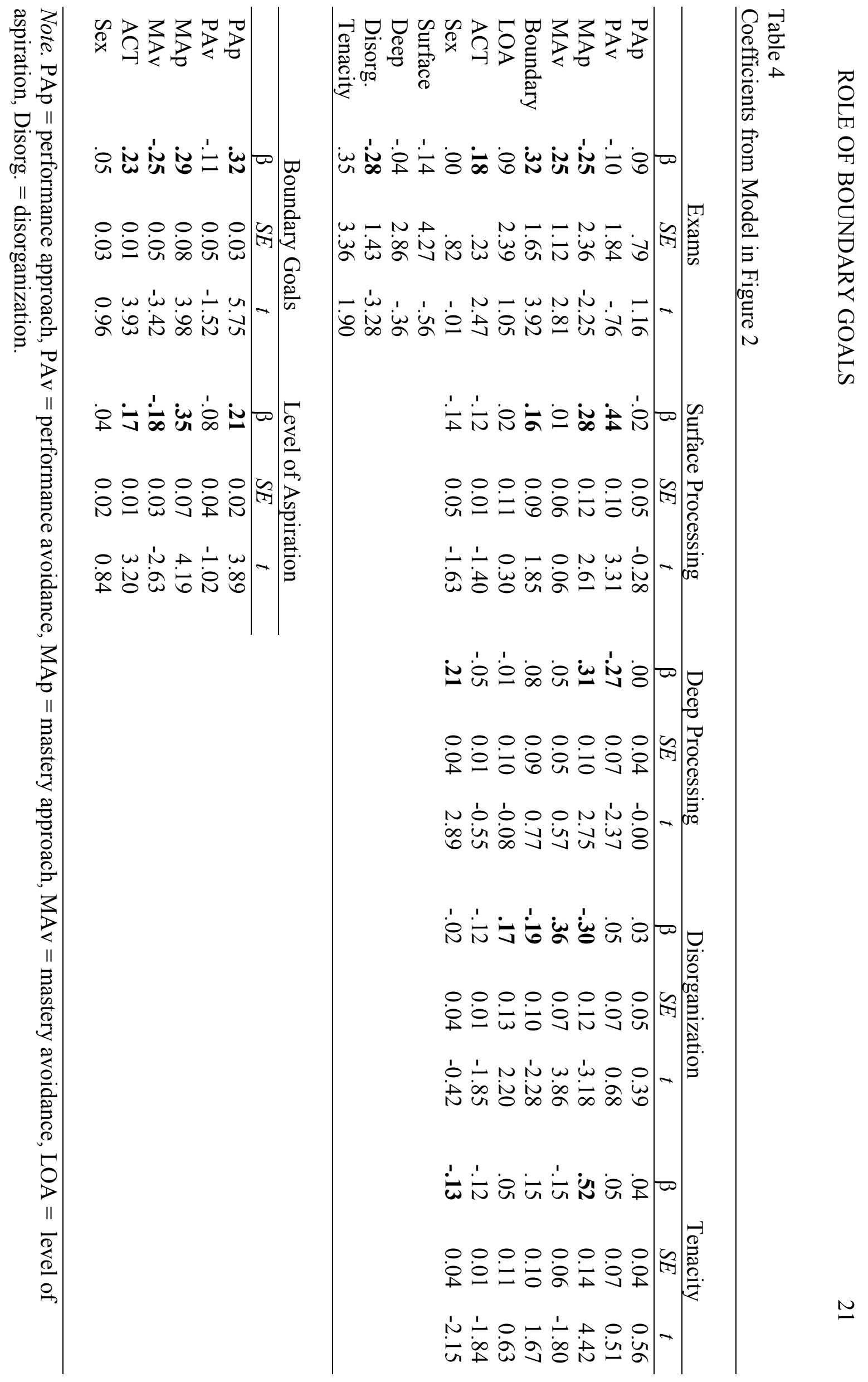



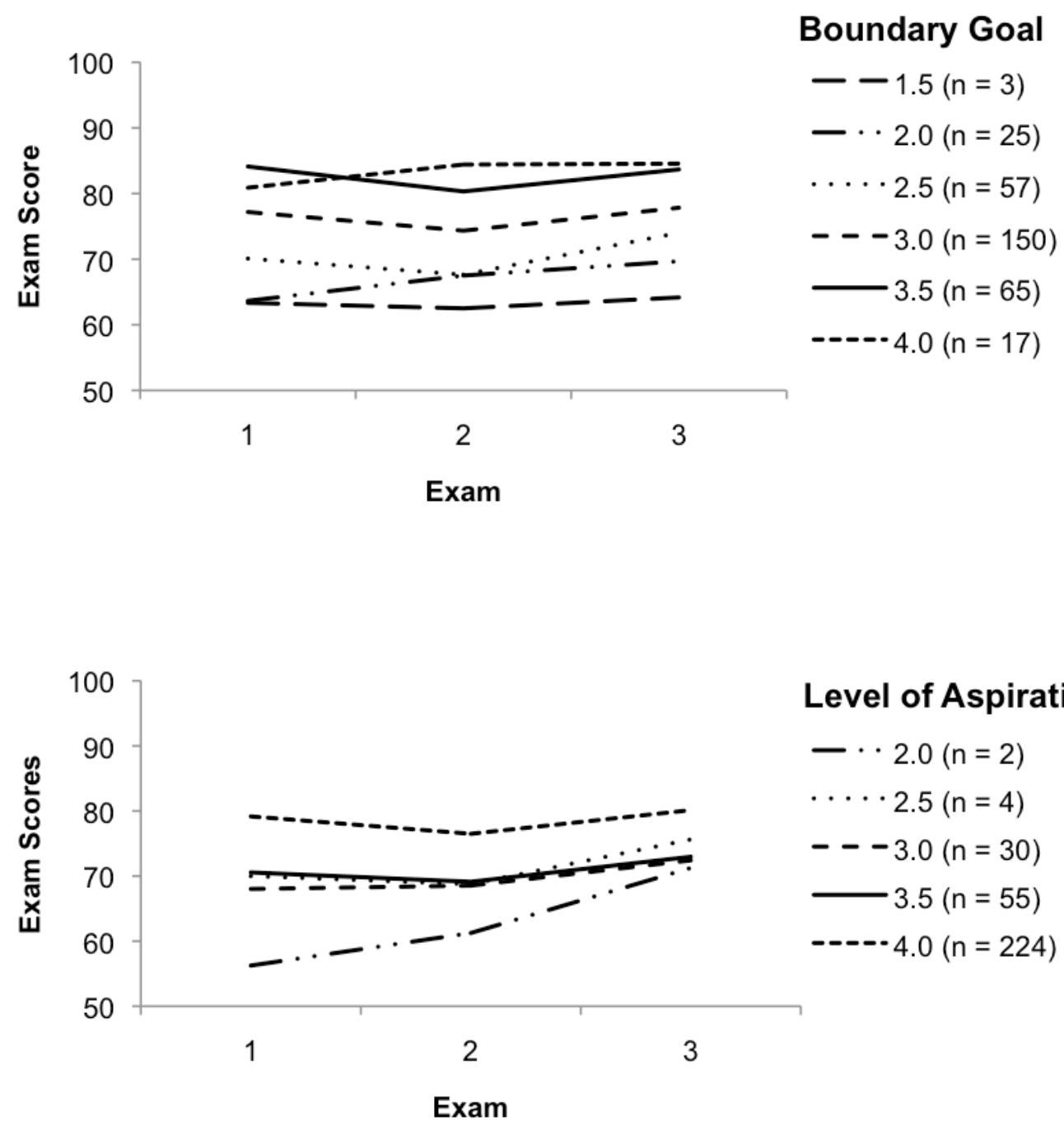

Figure 1. Average exam score trajectories of students with different boundary goal levels (top panel) and levels of aspriation (bottom panel) in grade point average units. 


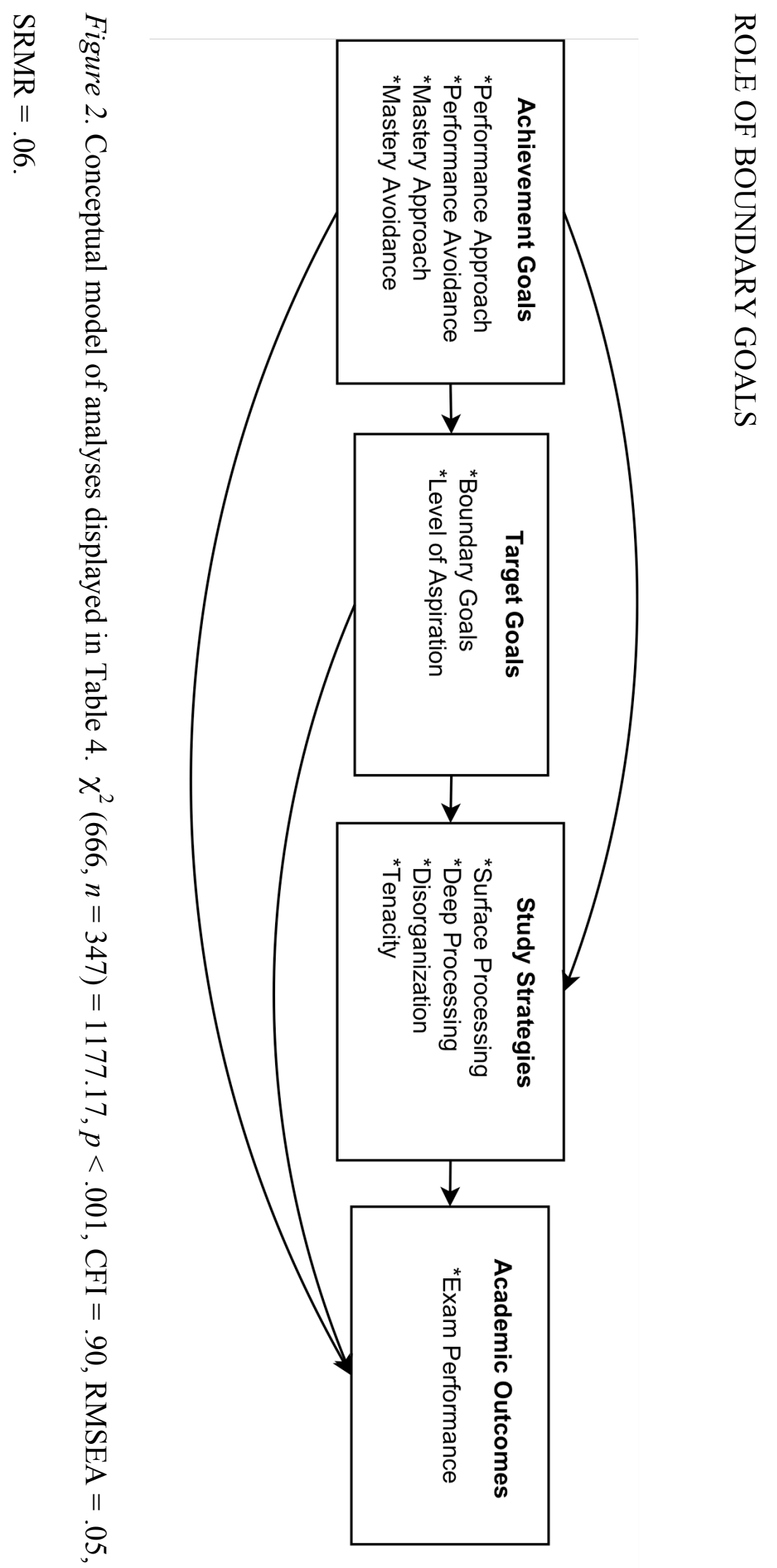

